\title{
Real-Time Hemodynamic Assessment of Downstream Effects of Intracranial Stenoses in Patients with Orthostatic Hypoperfusion Syndrome
}

\author{
Maher Saqqur ${ }^{a}$ Vijay K. Sharmab Georgios Tsivgoulisc, d Thang Nguyen Huy ${ }^{d}$ \\ loannis Heliopoulos ${ }^{c}$ Muzaffar Siddiqui ${ }^{\mathrm{a}}$ Carol Derksen ${ }^{\mathrm{a}}$ Khurshid Khan ${ }^{\mathrm{a}}$ \\ Andrei V. Alexandrov ${ }^{\mathrm{d}}$ \\ a Division of Neurology, Department of Medicine, University of Alberta, Edmonton, Alta., Canada; \\ ${ }^{b}$ Division of Neurology, Department of Medicine, National University Hospital, Singapore, Singapore; \\ 'Department of Neurology, Democrition University of Thrace School of Medicine, Alexandroupolis, Greece; \\ ${ }^{d}$ Comprehensive Stroke Center, University of Alabama, Birmingham Hospital, Birmingham, Ala., USA
}

\section{Key Words}

Intracranial arterial stenosis - Arterial flow velocity ·

Hypoperfusion

\begin{abstract}
Background: Arterial flow velocity changes on transcranial Doppler can reflect changes in cerebral flow during position-induced ischemia if obtained during short-term monitoring of positional changes. Subjects and Methods: Our multicenter group monitored symptomatic and asymptomatic arteries in patients with recurrent neurological deficits during positional changes and documented intracranial arterial stenosis. Bilateral posterior cerebral and middle cerebral arteries were monitored dependent on clinical symptom localization. The symptomatic artery was monitored distal to the intracranial stenosis, and mean flow velocities (MFV) were recorded at different body positions. The symptomatic artery relative MFV ratio was defined as the ratio of symptomatic artery MFV in the asymptomatic position MFV in the symptomatic position/MFV in the asymptomatic
\end{abstract}

position. Results: Sixteen patients underwent transcranial Doppler monitoring: mean age $62 \pm 19$ years, 11 (69\%) men, $6(40 \%)$ with transient ischemic attacks. Ten patients (63\%) had posterior and 6 anterior circulation symptoms. Patients developed neurological symptoms while standing up (63\%) and/or sitting (44\%), walking (13\%) or during neck extension (6\%). Symptomatic artery MFV dropped by $\geq 25 \%$ from the resting to the symptomatic position in all patients except for one. The mean symptomatic artery MFV relative ratio was higher compared with the mean asymptomatic artery MFV relative ratio: $0.5 \pm 0.28$ versus $-0.02 \pm 0.1(p=0.001$, Wilcoxon test). The symptomatic artery relative ratio of $>0.25$ had a $94 \%$ sensitivity and $100 \%$ specificity for predicting neurological symptom development during testing $(\kappa=0.9$, $p<0.001)$. Conclusions: A significant reduction in intracranial flow velocity distal to an intracranial stenosis can identify patients whose symptoms can worsen with positional changes. These patients may prove a target for interventional revascularization techniques.

Copyright $\odot 2010$ S. Karger AG, Basel

Maher Saqqur, MD, FRCPC

Division of Neurology, University of Alberta Hospital

2E3 Walter McKenzie Center 8440-112 St.

Edmonton, AB T6G 2B7 (Canada)

Tel. +1 780407 7574, Fax +1 780407 1325, E-Mail maher.saqqur@ ualberta.ca 


\section{Introduction}

Intracranial atherosclerotic stenosis is the culprit behind at least $8 \%$ of all ischemic stroke subtypes $[1,2]$. Subsequent infarctions secondary to intracranial atherosclerotic stenosis result from either flow failure known as hypoperfusion syndrome or artery-to-artery embolism [3].

The hypoperfusion cerebral transient ischemic attack (TIA) is uncommon and rarely reported in the literature. These TIAs typically occur with large artery hemodynamic stenosis in the internal carotid artery or in vertebrobasilar arteries resulting in a distal low-perfusion state and exhausted autoregulation with compromised vasomotor reserve [4-8].

Several conventional techniques have been used to evaluate the presence of stenotic disease in the anterior and posterior circulation like cerebral angiography, carotid ultrasound, CT and magnetic resonance angiography $[9,10]$.

In contrast to these conventional techniques, transcranial Doppler (TCD) may provide a dynamic real-time tool for quantitative measurements of arterial flow velocity changes that reflect changes in cerebral blood flow (CBF) during orthostatic or position-induced ischemia if obtained at a constant angle of insonation and during short-term monitoring of positional changes [11].

The objective of our study is to derive TCD monitoring criteria for hypoperfusion TIA syndrome in a series of patients with presumptive clinical hypoperfusion TIA caused by intracranial critical arterial stenosis or mechanical vascular compression with positional changes.

\section{Subjects and Methods}

We performed a retrospective collection of case series from multiple tertiary care university centers in North America, Europe and Asia of patients with documented intracranial severe or critical arterial stenosis or with mechanical vascular compression and recurrent neurological deficits during body or head positional changes. Verbal consent was taken from patients for potential research publication.

Cases of acute or subacute hypoperfusion TIA or stroke were defined as acute focal neurological symptoms that were induced by positional changing and caused by intracranial hemodynamic arterial stenosis or mechanical extracranial vascular compression confirmed on cerebral angiography or other noninvasive vascular imaging modalities (computed tomographic angiography or magnetic resonance angiography). All patients had neurological symptoms more than once, induced by positional changes (standing up, sitting, walking or during neck extension). Patients with a single neurological event were not included in our study population since the mechanism of their TIA was not clear.
Diagnostic TCD was performed at the bedside to detect an intracranial arterial stenosis or occlusion, and continuous TCD monitoring of the presumptively symptomatic artery was performed. In the presence of posterior circulation symptoms, bilateral posterior cerebral arteries (PCAs) through the temporal window or the basilar artery through the foramen magnum window were monitored. In the presence of hemispheric symptoms, the affected middle cerebral artery (MCA) was monitored distal to the intracranial stenosis/occlusion site dependent on the localization of clinical symptoms. In the presence of an anterior circulation stroke, the unaffected MCA was used for comparison, whereas in patients with posterior circulation symptoms, either the MCA or internal carotid artery was used for comparison. The TCD monitoring of affected and unaffected arteries was performed either simultaneously using a standard bilateral head frame or in sequence with only 1 transducer at a time positioned at a fixed angle. Finally, a 1-hour TCD emboli monitoring of the affected artery distal to the intracranial stenosis was performed to rule out concurrent microembolic activity.

The mean flow velocity (MFV) and pulsatility index (PI) of the affected and unaffected arteries at baseline in the resting position (supine) and in 5-10 min of being in the symptomatic position (sitting, standing or neck extension) were recorded and analyzed. An equilibration period of 5-10 min was instituted between measurements to ensure stability of both heart rate and arterial pressure to avoid a confounding influence on MFV.

The symptomatic artery relative MFV ratio was defined as follows: the ratio of affected artery MFV in the asymptomatic position - MFV in the symptomatic position/MFV in the asymptomatic position.

The unaffected artery relative MFV ratio was defined as follows: the ratio of unaffected (control) artery MFV in the asymptomatic position - MFV in the symptomatic position/MFV in the asymptomatic position.

Statistical analysis was performed using the $\chi^{2}$ test, Fisher's exact test, the unpaired t test, the paired t test, the Mann-Whitney $\mathrm{U}$ test and the Wilcoxon signed-rank test as indicated.

\section{Results}

Sixteen patients underwent TCD monitoring for possible acute or subacute positional TIA or stroke. The mean age was $62 \pm 19$ years, and 11 (69\%) were men. Six patients had TIA (40\%) and the rest had both TIA and stroke (60\%). Ten patients (63\%) had posterior and 6 (37\%) had anterior circulation symptoms. Patients developed neurological symptoms while standing up (37.5\%) and/or sitting (44\%), walking (12.5\%) or during neck extension (6\%) (table 1). Only 3 patients had angioplasty (2 with basilar artery stenosis and 1 with left MCA critical stenosis), 1 was treated with coumadin, 5 were treated with hydration, bed rest and antiplatelet agents (acetylsalicylic acid, plavix, aggrenox or acetylsalicylic acid and plavix) and the rest were treated with only antiplatelet agents. 
Table 1. Demographic data

\begin{tabular}{|c|c|c|c|c|c|c|}
\hline Patient & $\begin{array}{l}\text { Age } \\
\text { years }\end{array}$ & Sex & Symptoms & $\begin{array}{l}\text { Symptomatic } \\
\text { position }\end{array}$ & $\begin{array}{l}\text { Treatment at the } \\
\text { time of TCD }\end{array}$ & Vascular imaging (DSA, MRA or CTA) \\
\hline 1 & 56 & $\mathrm{M}$ & Broca's aphasia, right-arm weakness & standing & heparin + ASA & left terminal ICA MCA critical stenosis \\
\hline 2 & 50 & $\mathrm{M}$ & vertigo, ataxia & neck extension & ASA & normal $^{1}$ \\
\hline 3 & 74 & $\mathrm{~F}$ & Broca's aphasia, right-arm weakness & standing & ASA+plavix & left MCA severe stenosis \\
\hline 4 & 60 & M & Broca's aphasia, right-arm weakness & sanding & ASA & $\begin{array}{l}\text { left ICA occlusion, right terminal ICA severe } \\
\text { stenosis }\end{array}$ \\
\hline 5 & 80 & $\mathrm{~F}$ & arm weakness, dysarthria, vertigo & standing & none & severe right $\mathrm{VA}$ and proximal BA stenosis \\
\hline 6 & 68 & $\mathrm{M}$ & Broca's aphasia & sitting+VM & none & left MCA severe stenosis \\
\hline 7 & 74 & M & Broca's aphasia & sitting & ASA & left MCA severe stenosis \\
\hline 8 & 18 & M & right vision field cut, double vision, vertigo & standing & none & normal $^{2}$ \\
\hline 9 & 79 & M & arm and leg weakness, dysarthria, vertigo & sitting & ASA+plavix & severe BA stenosis \\
\hline 10 & 77 & $\mathrm{M}$ & arm and leg weakness, dysarthria, vertigo & standing & ASA+plavix & severe BA stenosis \\
\hline 11 & 76 & $\mathrm{~F}$ & dysarthria, left-arm weakness & sitting & plavix & right MCA severe stenosis \\
\hline 12 & 64 & M & dysarthria, dysphagia, ataxia & walking & aggrenox + ASA & $\begin{array}{l}\text { left VA occlusion and right VA moderate } \\
\text { stenosis }\end{array}$ \\
\hline 13 & 22 & $\mathrm{~F}$ & dysarthria, double vision, vision field cut & sitting & none & BA occlusion \\
\hline 14 & 64 & $\mathrm{M}$ & double vision, weakness & standing & ASA & right VA severe stenosis \\
\hline 15 & 56 & M & vision field cut, face and arm weakness & walking & plavix & BA occlusion \\
\hline 16 & 71 & $\mathrm{~F}$ & double vision, vertigo & sitting & ASA & BA severe stenosis \\
\hline
\end{tabular}

ASA = Acetylsalicylic acid; ICA = intracranial atherosclerotic; VA = vertebral artery; $\mathrm{BA}=$ basilar artery; $\mathrm{VM}=$ Valsalva maneuver.

${ }^{1}$ Patient No. 2 had large cervical anterior and posterior osteophytes that were causing mechanical compression of the left vertebral artery in the neck with the extension position. In addition, his right vertebral artery was hypoplastic on CT angiography.

${ }^{2}$ Patient No. 8 had meningitis and symptoms suggestive of spontaneous intracranial hypotension, but he developed focal symptoms referable to the left PCA territory while in hospital. Symptoms were reproducible with postural changes that likely induced a dominant vertebral artery obstruction. He received bed rest, hydration and a course of antibiotics, and symptoms could no longer be induced in 1 week.

Table 2. TCD flow findings

\begin{tabular}{|c|c|c|c|c|c|c|c|c|}
\hline Patient & $\begin{array}{l}\text { Affected } \\
\text { artery }\end{array}$ & MFV1 & MFV2 & $\begin{array}{l}\text { Affected } \\
\text { artery relative } \\
\text { ratio }\end{array}$ & $\begin{array}{l}\text { Unaffected } \\
\text { artery }\end{array}$ & MFV1 & MFV2 & $\begin{array}{l}\text { Unaffected } \\
\text { artery relative } \\
\text { ratio }\end{array}$ \\
\hline 1 & left MCA & 110 & 78 & 0.29 & right MCA & 46 & 40 & 0.13 \\
\hline 2 & bilateral PCA & 19 & 0 & 1.00 & left MCA & 48 & 48 & 0.00 \\
\hline 3 & left MCA & 68 & 42 & 0.38 & right MCA & 74 & 70 & 0.05 \\
\hline 4 & left MCA & 36 & 22 & 0.39 & right $\mathrm{MCA}$ & 66 & 71 & -0.08 \\
\hline 5 & $\mathrm{BA}$ & 42 & 19 & 0.55 & & & & \\
\hline 6 & left MCA & 56 & 38 & 0.32 & right MCA & 62 & 78 & -0.26 \\
\hline 7 & left MCA & 36 & 20 & 0.44 & right $\mathrm{MCA}$ & 56 & 56 & 0.00 \\
\hline 8 & left PCA & 46 & 30 & 0.35 & right PCA & 48 & 50 & -0.04 \\
\hline 9 & BA & 25 & 0 & 1.00 & & & & \\
\hline 10 & $\mathrm{BA}$ & 150 & 0 & 1.00 & & & & \\
\hline 11 & right MCA & 46 & 38 & 0.17 & left MCA & 66 & 68 & -0.03 \\
\hline 12 & right PCA & 17 & 0 & 1.00 & right MCA & 67 & 67 & 0.00 \\
\hline 13 & $\mathrm{BA}$ & 40 & 15 & 0.63 & right MCA & 46 & 39 & 0.15 \\
\hline 14 & right VA & 44 & 27 & 0.39 & right MCA & 47 & 49 & -0.04 \\
\hline 15 & $\mathrm{BA}$ & 36 & 21 & 0.42 & right MCA & 53 & 55 & -0.04 \\
\hline 16 & $\mathrm{BA}$ & 47 & 23 & 0.51 & right MCA & 61 & 69 & -0.13 \\
\hline
\end{tabular}

MFV1 = Mean flow velocity in the asymptomatic position; MFV2 = mean flow velocity in the symptomatic position; affected artery relative ratio $=$ ratio of affected artery MFV in the asymptomatic position $-\mathrm{MFV}$ in the symptomatic position/MFV in the asymptomatic position; unaffected artery relative ratio = ratio of unaffected (control) artery MFV in the asymptomatic position - MFV in the symptomatic position/MFV in the asymptomatic position; $\mathrm{BA}=$ basilar artery; VA = vertebral artery. 
a

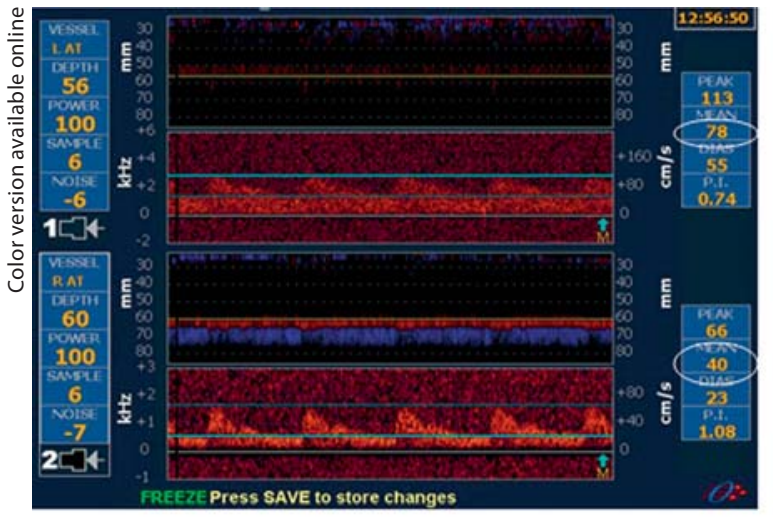

Cerebral angiography: left supraclinoid ICA critical stenosis

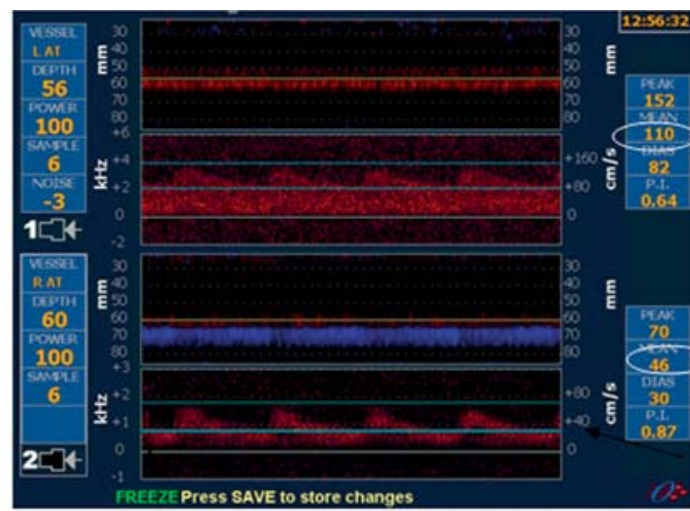

Affected left MCA

Contralateral MCA (control) b

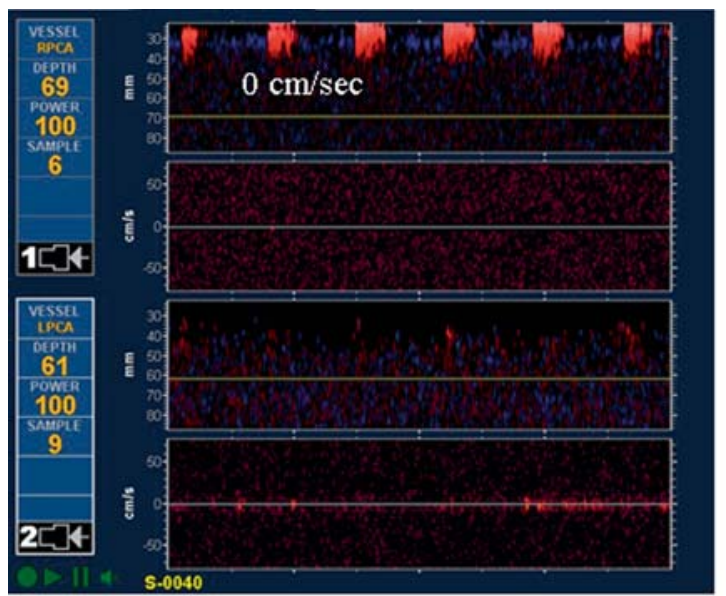

Lying down position

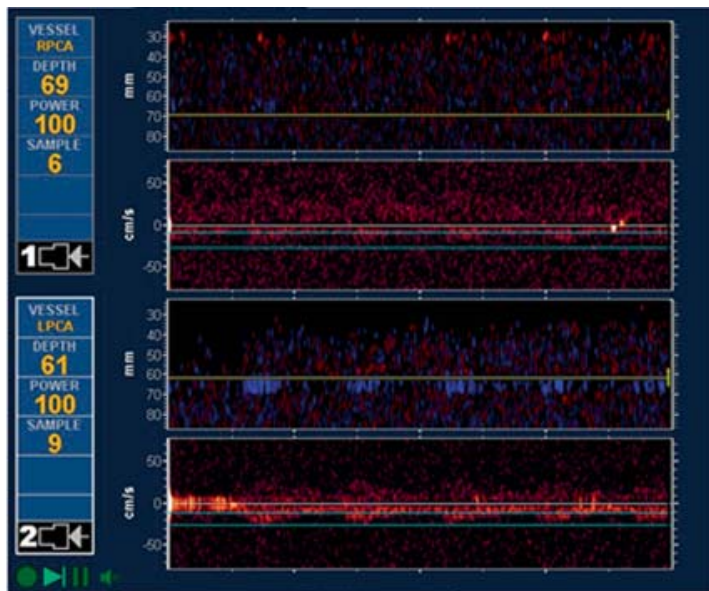

Left PCA:

MFV $17 \mathrm{~cm} / \mathrm{s}$

Right PCA

MFV $16 \mathrm{~cm} / \mathrm{s}$

Cerebral angiography: left VA occlusion and right VA severe stenosis

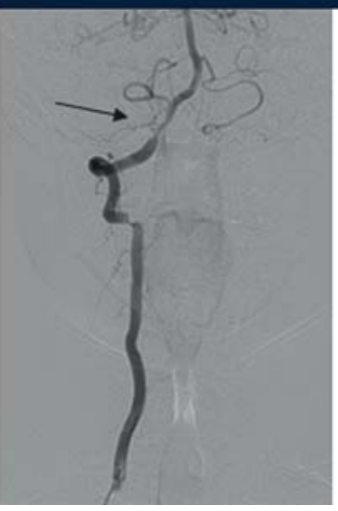




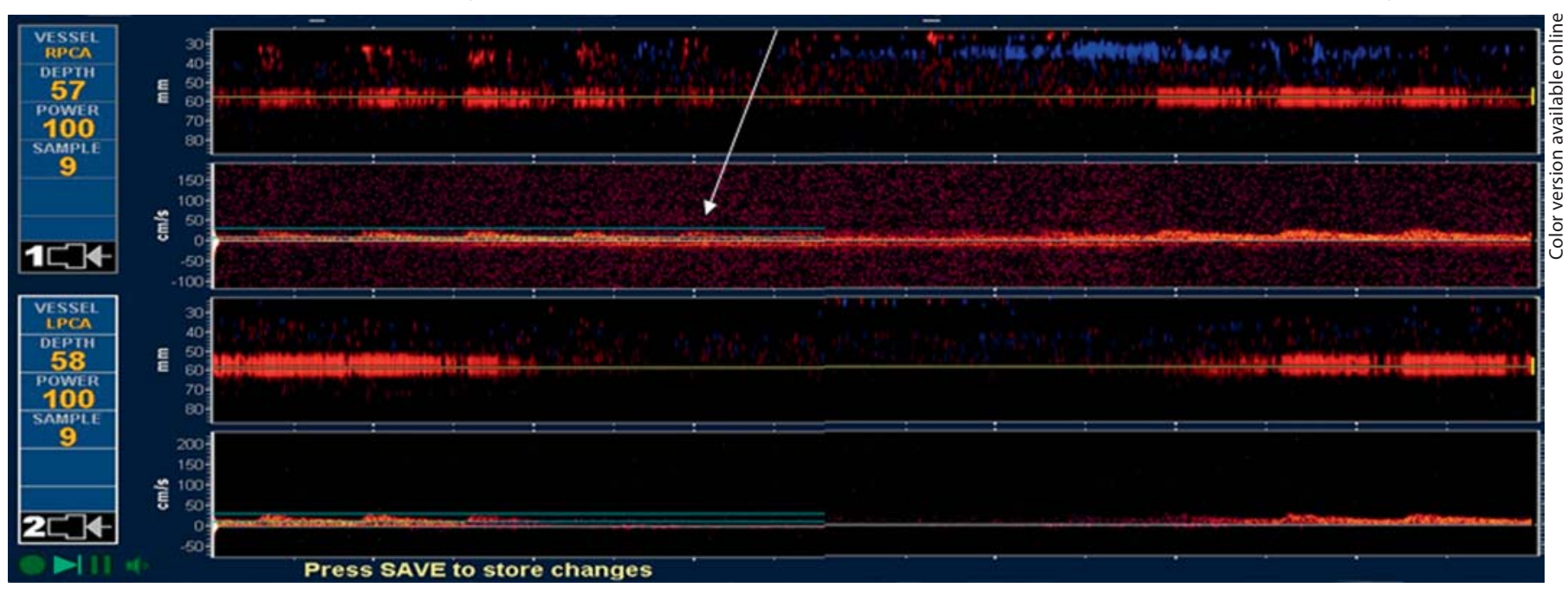

Fig. 1. An example of 3 patients with anterior and posterior circulation hypoperfusion TIA syndrome. a Anterior circulation hypoperfusion TIA: a 55-year-old man with orthostatic episodes of expressive aphasia and right-arm weakness. Digital subtraction angiography showed critical left supraclinoid internal carotid artery (ICA) stenosis. The affected MCA MFV dropped from 110 $\mathrm{cm} / \mathrm{s}$ in the lying down position to $78 \mathrm{~cm} / \mathrm{s}$ in the standing position. The affected artery MCA MFV relative ratio was 0.29 (11078/110) and the unaffected MCA relative MFV ratio was 0.13 (4640/40). b Posterior circulation hypoperfusion TIA: a 64-year-old man with orthostatic episodes of dysarthria, dysphasia and ataxia. Cerebral angiography revealed left vertebral artery (VA) occlusion and right vertebral artery stenosis. The affected PCA MFV dropped from $17 \mathrm{~cm} / \mathrm{s}$ in the lying flat position to $0 \mathrm{~cm} / \mathrm{s}$ in the sitting-up position. The affected artery PCA relative MFV ratio was 1 (17-0/17), and the unaffected (control) right MCA MFV relative ratio was 0 (67-67/67). c Posterior circulation hypoperfusion TIA: a 50-year-old man with transient episodes of ataxia and vertigo induced by neck extension. The patient had large cervical anterior and posterior osteophytes that were causing mechanical compression of the left vertebral artery in the neck. In addition, his right vertebral artery was hypoplastic on CT angiography. His TCD monitoring of bilateral PCAs revealed a drop in the MFV from 19 to $0 \mathrm{~cm} / \mathrm{s}$ with neck extension. The affected artery MFV relative ratio was $1(19-0 / 19)$ and the unaffected (control) left MCA MFV relative ratio was 0 (48-48/48).
TCD flow findings are summarized in table 2. The mean affected artery MFV in the resting position was 52 $\pm 34 \mathrm{~cm} / \mathrm{s}$. It did drop to $24.8 \pm 20 \mathrm{~cm} / \mathrm{s}$ in the symptomatic position ( $\mathrm{p}<0.001$, Wilcoxon test). The mean unaffected artery MFV was $57 \pm 10$ and $59 \pm 13 \mathrm{~cm} / \mathrm{s}$ in the resting and symptomatic positions, respectively $(\mathrm{p}=$ 0.37). Figure 1 shows 3 cases with MFV changes when symptoms were induced with different body positions.

The affected artery MFV dropped by $\geq 25 \%$ from resting to the symptomatic position in all patients except for one (drop by $17 \%$ ). The mean MFV relative ratio of the affected arteries was higher compared with the mean MFV relative ratio of the unaffected arteries: $0.5 \pm 0.28$ versus $-0.02 \pm 0.1(\mathrm{p}=0.001$, Wilcoxon test). The affected artery relative MFV ratio of $>0.25$ had a $94 \%$ sensitivity, $100 \%$ specificity, $100 \%$ positive predictive value and $93 \%$ negative predictive value for predicting neurological symptom development during testing ( $\kappa$ test for agreement $0.9 ; \mathrm{p}<0.001)$.

TCD Monitoring Criteria for Hypoperfusion TIA Syndrome
The mean affected artery PI in the resting and symptomatic positions was $0.8 \pm 0.25$ and $0.88 \pm 0.48$, respectively ( $\mathrm{p}=0.29$, Wilcoxon test). The mean unaffected artery $\mathrm{PI}$ in the resting and symptomatic positions was 0.88 \pm 0.08 and $0.89 \pm 0.12(\mathrm{p}=0.68$, Wilcoxon test). In addition, the mean drop in PI in the symptomatic artery and the unaffected artery was $0.15 \pm 0.49$ and $0.008 \pm$ 0.12 , respectively $(\mathrm{p}=0.15)$. No microembolic activity was detected during the TCD emboli monitoring.

\section{Discussion}

Our study showed that the affected artery relative MFV ratio in the symptomatic position corresponds to the development of clinical symptoms and could be helpful in identifying patients with hypoperfusion TIA syndrome. The orthostasis-induced flow changes and clinical symptoms provide a study population suitable to test

Cerebrovasc Dis 2010;30:355-361 
these findings prospectively. If further independent studies validate our newly developed criteria, the dynamic TCD technique may enhance the recognition of patients with imminent hypoperfusion symptoms and triage them to a more definitive therapy. The management of hypoperfusion TIA is still controversial - between an interventional stenting treatment of the stenotic artery and just blood pressure management. Future trials using TCD as an adjunctive diagnostic tool are warranted.

The exact mechanism of hypoperfusion TIA is unknown. The most accepted hypothesis is that transient cerebral ischemia results from a low-perfusion hemodynamic state due to severe extracranial and/or intracranial carotid or MCA or vertebrobasilar arterial disease [12-14]. In general orthostatic position changes, the longstanding position, neck extension or hypotension induced by antihypertensive medication have been reported to trigger hypoperfusion TIA, thus suggesting the lowperfusion hemodynamic mechanism $[15,16]$. Any temporal reduction in $\mathrm{CBF}$ can result in a cerebral ischemia which could manifest in several ways like limb-shaking TIA or brief stereotyped orthostatic focal neurological symptoms $[17,18]$.

On a cellular level, the chronic hypoperfusion state produces lactic acidosis that in turn leads to arterial vasodilatation in order to maintain CBF in the hypoperfusion tissue. Note that the low PI values documented at rest are due to reducing inflow because of the intracranial arterial stenosis, and the lack of response in flow pulsatility despite a significant velocity reduction may be attributed to lower impedance of collateral vessels in the symptomatic position [19]. Further studies are needed to better understand this phenomenon where TCD and perfusion brain MRI can be performed in sequence. Finally, the lack of PI changes in the unaffected vessels parallels no change in flow velocities that eliminated the need for additional vasodilation as perfusion conditions were largely unchanged in the patent vessels.

Several neuroimaging modalities have been used to evaluate $\mathrm{CBF}$ of the brain in patients with hypoperfusion TIAs. Xenon-133 inhalation, xenon-enhanced CT scanning, single photon emission computed tomography scan and positron emission tomography with fluorodeoxyglucose have been used to determine regional $\mathrm{CBF}[7,20]$. TCD is a noninvasive and inexpensive monitoring tool that can be performed at the bedside to assess the hemodynamic status of cerebral arteries in different body positions as we demonstrated in our study. It can become a critical part in confirming the etiology of hypoperfusion TIA syndrome by suggesting a drop in blood flow rather than being an embolic phenomenon in the presence of critical arterial stenosis. In addition, there is a potential role for TCD monitoring in selecting further treatment and follow-up. This issue has not been addressed in the present study and we are planning to investigate this prospectively.

One of the main limitation of TCD is that it measures blood flow velocities and not the actual cerebral perfusion flow in the brain tissues. However, the intracranial flow velocity can reflect $\mathrm{CBF}$ and the velocity changes can be proportional to changes in CBF tissue as long as the following factors are taken into consideration during the TCD monitoring: the angle of insonation remains constant, the perfusion territory remains the same, and the effect of only 1 stimulus is observed [21]. In our study, we tried to account for these factors in all cases during the TCD monitoring. Another limitation of this study is related to the fact that TCD is still operator dependent and requires specialized training for application in the stroke setting. This was addressed by ensuring that all sonographers were trained and certified in TCD skills pertinent to the stroke setting. In addition, the head frame may not be deployed in all cases of posterior symptoms, so the hand-held MFV measurements could be suboptimal. Finally, we could not find a correlation between TCD parameters and blood pressure measurements in our study patients. In addition, different types of collateral blood flow on cerebral angiography were not provided since our study was a retrospective one, and this correlation was not put in our a prior hypothesis.

In conclusion, dynamic TCD monitoring may be helpful in identifying TIAs or strokes due to hypoperfusion in patients with steno-occlusive intracranial arterial disease. Further studies are needed to validate our TCD findings in a heterogeneous group of patients with symptomatic and asymptomatic intracranial arterial stenosis.

\section{Disclosure Statement}

A.V. Alexandrov received research grant (USD >10,000) NINDS K 23-02229. Speaker bureau for Genentech, Honoraria (USD < 10,000) from Genentech. 


\section{References}

1 Warlow CP, Morris PJ: Transient Ischemic Attacks. New York, Marcel Dekker, 1982.

-2 Ruff RL, Talman WT, Petito F: Transient ischemic attacks associated with hypotension in hypertensive patients with carotid artery stenosis. Stroke 1981;12:353-355.

-3 Caplan LR, Wong KS, Gao S, Hennerici MG: Is hypoperfusion an important cause of strokes? If so, how? Cerebrovasc Dis 2006;21: 145-153.

4 Somerville ER: Orthostatic transient ischemic attacks: a symptom of large vessel occlusion. Stroke 1984;15:1066-1067.

$\checkmark 5$ Pascual-Leone A, Anderson DC, Larson DA: Volume therapy in orthostatic transient ischemic attacks. Stroke 1989;20:1267-1270.

-6 Baquis GD, Pessin MS, Scott RM: Limb shaking - a carotid TIA. Stroke 1985;16: 444-448.

7 Tatemichi TK, Young WL, Prohovnik I, Gitelman DR, Correll JW, Mohr JP: Perfusion insufficiency in limb-shaking transient ischemic attacks. Stroke 1990;21:341-347.

-8 Alexandrov AV, Sharma VK, Lao AY, Tsivgoulis G, Malkoff MD, Alexandrov AW: Reversed Robin Hood syndrome in acute ischemic stroke patients. Stroke 2007;38: 3045-3048.

\9 Nguyen-Huynh MN, Wintermark M, English J, Lam J, Vittinghoff E, Smith WS, Johnston SC: How accurate is CT angiography in evaluating intracranial atherosclerotic disease? Stroke 2008;39:1184-1188.
10 Feldmann E, Wilterdink JL, Kosinski A, Lynn M, Chimowitz MI, Sarafin J, Smith HH, Nichols F, Rogg J, Cloft HJ, Wechsler L, Saver J, Levine SR, Tegeler C, Adams R, Sloan $M$, The Stroke Outcomes and Neuroimaging of Intracranial Atherosclerosis (SONIA) Trial Investigators: The Stroke Outcomes and Neuroimaging of Intracranial Atherosclerosis (SONIA) trial. Neurology 2007;68:20992106.

11 Newell DW, Aaslid R: Transcranial Doppler: clinical and experimental uses. Cerebrovasc Brain Metab Rev 1992:4:122-143.

12 Caplan LR, Wityk RJ, Glass TA, Tapia J, Pazdera L, Chang HM, Teal P, Dashe JF, Chaves CJ, Breen JC, Vemmos K, Amarenco P, Tettenborn B, Leary M, Estol C, Dewitt LD, Pessin MS: New England Medical Center Posterior Circulation registry. Ann Neurol 2004;56:389-398.

13 Tatemichi TK, Young WL, Prohovnik I, Gitelman DR, Correll JW, Mohr JP: Perfusion insufficiency in limb-shaking transient ischemic attacks. Stroke 1990;21:341-347.

14 Jiang W, Gao F, Du B, Srivastava T, Wang Y: Limb-shaking transient ischemic attack induced by middle cerebral artery stenosis. Cerebrovasc Dis 2006;21:421-422.

15 Dobkin BH: Orthostatic hypotension as a risk factor for symptomatic occlusive cerebrovascular disease. Neurology 1989;39:3034
16 Khan A, Beletsky V, Kelley R, Ehsan T: Orthostatic-mediated hypoperfusion in limbshaking transient ischemic attack. J Neuroimaging 1999;9:43-44.

17 Yonas H, Pindzola RR: Physiological determination of cerebrovascular reserves and its use in clinical management. Cerebrovasc Brain Metab Rev 1994;6:325-340.

18 Yonas H, Smith HA, Durham SR, Pentheny SL, Johnson DW: Increased stroke risk predicted by compromised cerebral blood flow reactivity. J Neurosurg 1993;79:483-489.

-19 Schneider P, Rossman M, Bernstein E, Torem S, Ringelstein E, Otis S: Effect of internal carotid artery occlusion on intracranial hemodynamics: transcranial Doppler evaluation and clinical correlation. Stroke 1988; 19:589593.

20 Martí-Fàbregas JA, Catafau AM, Marí C, Mendoza G, Sanahuja J, Lleó A, Martí-Vilalta JL: Cerebral perfusion and haemodynamics measured by SPET in symptom-free patients with transient ischaemic attack: clinical implications. Eur J Nucl Med 2001; 28:1828-1835.

21 Newell DW, Aaslid R, lam A, Mayberg TS, Winn HR: Comparison of flow and velocity during dynamic auto-regulation in humans. Stroke 1994;25:793-797. 